\title{
Mechanism-Oriented Therapy of Irritable Bowel Syndrome
}

Juan R. Malagelada · Carolina Malagelada

Received: February 24, 2016/Published online: April 30, 2016

(c) The Author(s) 2016. This article is published with open access at Springerlink.com

\section{ABSTRACT}

Irritable bowel syndrome (IBS) is a common and well-accepted diagnosis but often imprecisely applied to patients in usual clinical practice. Diagnosis is entirely based on symptom criteria that tend to include broad strata of abdominal complainers. Established criteria for diagnosis are strictly followed in controlled clinical trials for new therapeutic agents, but physicians are more lax in the clinic. Predictably, in light of the above ambiguities, many pathogenetic mechanisms and pathophysiological disturbances appear to be involved in IBS, but so far no mechanism-based subgroupings to guide specific therapy have been soundly established. Thus, diverse therapeutic approaches coexist and are discretionally prescribed by attending clinicians on the basis of major manifestations (i.e., diarrhea-predominance or constipation-predominance), more or less apparent psychological disturbances, and patient

Enhanced content To view enhanced content for this article go to http://www.medengine.com/Redeem/13C4 F0607800E00A.

J. R. Malagelada ( $₫) \cdot$ C. Malagelada

Digestive System Research Unit, University Hospital

Vall d'Hebron, Barcelona, Spain

e-mail: jrmalagelada@vhebron.net preferences (pharmacological versus dietary or microbiological approaches). In this review, we have attempted to update scientific knowledge about the more relevant disease mechanisms involved and relate this more fundamental basis to the various treatment options available today.

Keywords: Gastroenterology; Irritable bowel syndrome; Mechanism; Pharmacology; Treatment

\section{GENERAL CONSIDERATIONS}

In Western countries, irritable bowel syndrome (IBS) is a common medical condition with prevalence figures that hover around 10-15\% [1]. Other countries around the world also report high prevalence figures, albeit more variable. IBS also constitutes a substantial fraction of specialist consultations that, in the USA, has been estimated to be close to $20 \%$ [2]. IBS impacts considerably on a patient's daily living and quality of life while increasing healthcare resource use and expenditure. Consequently, IBS results in a substantial financial and economic burden including costs 
for consultations, diagnostic tests, medications, and preventive measures together with substantial productivity losses.

IBS is a term that currently enjoys favor with medical professionals and it is also increasingly recognized as a valid diagnosis by patients. However, the definition and especially the scope of IBS as a medical condition remain somewhat imprecise because its diagnosis is entirely symptom-based and there are no biomarkers or diagnostic technologies available for precise characterization [3, 4]. Since 1992, there have been four major consecutive attempts at defining IBS by consensus during medical expert meetings in Rome, the last being held in 2015. The successively refined definition of IBS that has emerged from these Rome meetings has been helpful in popularizing the term IBS and providing concrete symptom criteria. In turn, these established criteria have enabled regulatory agencies to standardize the requirements for the performance of randomized control trials, subsequently conducted by pharma industries striving to obtain approval for new drugs intended for treatment of IBS. From the above perspective, the Rome process has yielded generally accepted outcomes and proven value. However, problems remain with the exclusively symptom-based definition of IBS that risks mixing pathophysiologies and possibly even etiologies within trial study groups. Furthermore, concerns have been expressed about the difficulties that would be encountered in the future to disentangle the current terminology should new methods be developed to establish the diagnosis beyond exclusive symptom criteria. Furthermore, as pointed out by Quigley and Shanahan [5], an exclusively symptom-based definition of a medical condition such as IBS and other functional syndromes may unintentionally create an illusion of understanding and equate complaints with "disease". Such risk is already noticeable in clinical practice as physicians often include cases of unexplained abdominal pain into the IBS diagnosis without specifically checking whether all these patients really meet published Rome criteria [6].

Another important aspect of IBS diagnosis, which is highly relevant to therapeutic strategies, is the fact that patients with IBS often manifest associated extraintestinal symptoms and comorbid conditions. Among the most common are fatigue, musculoskeletal pain, headaches, sleep disturbance, urinary symptoms, and comorbidities such as fibromyalgia, chronic fatigue syndrome, intestinal cystitis, sicca syndrome, post-traumatic stress disorder, temporomandibular joint disorder, and chronic pelvic pain [7]. These associated symptoms and comorbidities raise the following question: are we dealing with a gastroenterological or a systemic medical problem? Indeed, as reasoned by Ross [8], there may be an element of "meme" contagion fuelling the astonishingly high prevalence of IBS symptoms in many Western societies. The IBS "meme" perspective implies that the IBS label itself becomes a useful and compelling idea capable of inducing psychosomatic illness in vulnerable "hosts". Furthermore, symptoms themselves may be promoted and amplified by medical professionals eager to establish a diagnosis.

This article is based on previously conducted studies and does not involve any new studies of human or animal subjects performed by any of the authors. 


\section{APPROACHING IBS THERAPY FROM A PATHOPHYSIOLOGICAL PERSPECTIVE}

Any therapeutic approach to IBS should contemplate the aspects outlined in Table 1, including epidemiological data applicable to an individual patient, the specific symptomatology and severity of illness, the associated manifestations, and comorbidities that may be present, and the personality traits and psychosomatic aspects, which cannot be ignored. These various features may provide useful hints about the pathogenetic mechanisms operating in a given patient, helping us direct the various potential therapeutic measures in the most efficient way. Let us consider next the most relevant aspects of such a discriminating approach.

\section{Immunological Gut Dysregulation}

There is mounting evidence of altered gut mucosal immune activity [9-11] including the presence of mucosal immune cell infiltrates [12], modified mucosal lymphocyte phenotypes [13], mast cell proliferation in proximity to nerve endings [14], and increased apical junction complex permeability [15]. Furthermore, elevated levels of circulating proinflammatory cytokines have been reported [16]. There is also indication of microbial induced mucosal immune activity based on observations such as increased blood antibodies against flagellin and increased antimicrobial peptides such as B2 defensins. In certain models there is evidence that probiotics (i.e., Bifidobacterium infantis, Faecalibacterium prausnitzii) may diminish proinflammatory

Table 1 Known IBS pathophysiologic disturbances and suitable as therapeutic targets

\begin{tabular}{ll}
\hline Disturbances & Targets \\
\hline Immunological gut dysregulation & Mucosal inflammation \\
& Neuroimmune interactions \\
Altered microbial gut ecology & SIBO \\
& Excess bowel fermentation (carbohydrates, protein) \\
& Short chain fatty acid production \\
Diet composition and tolerance & Lactose, fructose, and gluten intolerance \\
& FODMAP's symptom induction \\
& Prebiotic/symbiotic action \\
Brain-gut axis dysfunction & Food allergens \\
& Visceral hypersensitivity \\
Bile acid malabsorption & Extraintestinal manifestations \\
Increased bile acid synthesis & Stress-induced CNS, ENS, HPA axis dysfunctions \\
\hline
\end{tabular}

$C N S$ central nervous system, ENS enteric nervous system, HPA hypothalamic-pituitary-adrenal, SIBO small intestine bacterial overgrowth, FODMAP fermentable oligo-, di-, mono-saccharides and polyols 
cytokine activity (NF- $\kappa \mathrm{B}$ and IL-8) providing some mechanistic support for the therapeutic use of probiotics in IBS.

\section{Microbial Gut Ecology}

Post-infectious IBS is a special form where mucosal inflammation and abnormal gut-host microbial interactions probably play a particularly significant role [17]. Epidemiological data indicates that post-infectious IBS may affect from 3\% to 30\% of individuals developing infectious gastroenteritis [18]. Predisposing factors include female sex, younger age, prior antibiotics, and concomitant depression. Interestingly, psychological comorbidity increases the risk of developing IBS via enhanced susceptibility to develop infectious gastroenteritis [19]. In post-infectious IBS, mucosal immune activation and immune cell proliferation may enhance peripheral sensory signaling [20] and contribute to visceral hypersensitivity which is considered a pivotal symptom mechanism in IBS.

Microbiota alterations may also contribute to the pathogenesis of IBS, but evidence so far is spotty and sometimes contradictory. A potential IBS disease mechanism involving microbiota alterations is microbial proliferation outside the main bacterial reservoir, which is the so-called small intestine bacterial overgrowth (SIBO) hypothesis. This potential mechanism was initially proposed by Pimentel et al. [21] and others about 20 years ago. The concept of SIBO chiefly emanated from reports of frequently positive lactulose $\mathrm{H}_{2}$ breath tests in patients with IBS. However, other studies could find no difference between diarrhea-predominant IBS (IBS-D), constipation-predominant IBS (IBS-C), and controls [22, 23], and data from culture of jejunal aspirates proved inconclusive. It was further argued that jejunal aspirates were not representative of the distal small bowel luminal environment where bacterial overgrowth might be more significant and also that the composition of the small bowel microbiota differed between IBS and healthy controls. In any case, the hypothesis that SIBO was a relevant symptom mechanism in IBS was underscored by the plausibility of a number of putative mechanisms: small bowel deconjugation of bile acids with liberation of diarrheagenic moieties, generation of short chain fatty acids and gases with increased 5HT release and stimulation of ileal contractions, endotoxin-induced hyperalgesia, and production of a mucosal low grade inflammatory state with increased visceral hypersensitivity [24]. Indeed, some data suggest correlation between fecal short chain fatty acid content and IBS symptoms. Protein fermentation in the small bowel could also lead to increased production of hydrogen sulfides that may alter epithelial metabolism and induce visceral hypersensitivity. On the other hand, the potential relevance of SIBO was substantially weakened by the studies of $\mathrm{Yu}$ et al. [25] that combined orocecal scintigraphic measurements of transit with the conventional lactulose $\mathrm{H}_{2}$ breath test. These investigators evaluated 40 patients with IBS whose breath tests were positive (applying conventional criteria) and showed that $88 \%$ of these patients had at least $5 \%$ of the radioactive tracer in the cecum at the time of the $\mathrm{H}_{2}$ breath ascent. Thus, although this comparative study does not exclude the possibility of SIBO, it shows that breath test positivity is not a reliable criterion since rapid transit of marker from mouth to colon probably explains the fallaciously high proportion of patients with IBS so diagnosed. 
Alternative explanations to SIBO may account for the symptomatic responses to antibiotic treatment in IBS observed in some studies. Such alternative explanations involve alterations in fecal microbiota composition that have been observed in patients with IBS. These include qualitative and quantitative changes in some bacterial families [26]. Interestingly, certain human microbiota species appear to influence specific and relevant gut functions. For instance, F. prausnitzii may operate as a modulator of colonic hypersensitivity [27] and Lactobacillus reuteri as a regulator of colonic transit [28]. Unfortunately, progress in this area is hampered by the absence of good biomarkers of healthy microbiota to predict disease or to help us monitor responses to agents acting of human gut microbiota. Future research may help clarify such aspects.

\section{Diet Composition and Tolerance}

Lactose intolerance relates to lactase nonpersistence which may affect up to $65 \%$ of the adult population [29]. True lactose intolerance may induce IBS-like symptoms but only with relatively high lactose loads ( $>20 \mathrm{~g}$ ) that are easily avoidable by forewarned patients. On the other hand, psychological factors have a major influence on the symptomatic responses to lactose intake [30].

Fructose is a monosaccharide, abundantly present in many processed foods. The human small bowel has a relatively limited absorptive capacity that particularly affects free fructose which is the fraction in excess of the glucose that facilitates fructose absorption [31]. Thus, high fructose loads may induce symptoms even in healthy individuals. Fructans and galactooligosaccharides are poorly absorbed short chain carbohydrates that may reach the colon intact, increasing colonic production of gas via fermentation. Polyols comprising sorbitol, mannitol, and xylitol are also naturally present in many fruits and vegetables as well as added as artificial sweeteners to industrial food products and pharmaceuticals. They tend to induce bowel discharges because of their stimulant effect on intestinal motility [32]. The aforementioned short chain fermentable carbohydrates are collectively termed FODMAPs, and there is direct evidence by magnetic resonance imaging technology that among FODMAPs there are some, like fructose, that may induce symptoms via increased small bowel water content whereas others, like fructans, increase colonic gas production [33].

Heightened awareness of the role of gluten sensitivity in the pathogenesis of celiac disease has quickly evolved into the concept of "gluten intolerance" portrayed as an IBS-like condition clinically manageable by dietary gluten restriction. This "gluten-free healthy diet" concept has rapidly spread to the fashion and media community and from them to the general population where it has acquired many adepts. The marketing of gluten-free food products has rapidly expanded in suit. Uncertainties as to the pathogenesis of the phenomenon and the actual therapeutic value of gluten avoidance (total or partial) in these individuals are still the subject of controversy.

Prebiotics are food products that favor proliferation of bifidobacteria and other species potentially associated with anti-inflammatory effects (oligofructose, inulin, galactooligosaccharides, lactulose, breast milk oligosaccharides, and others). Prebiotics do not seem particularly effective in IBS possibly on account of fermentation products that may, in themselves, stimulate symptoms. The so-called synbiotics that aim to 
simultaneously produce synergic pro- and prebiotic effects also have not encountered much therapeutic success in IBS.

Specific components of the normal diet have long been suspected to induce gastrointestinal symptoms in susceptible individuals. In fact, patients themselves often insist that they may experience symptomatic responses to concrete foods. This general concept of food intolerance being the origin of IBS-like symptoms is appealing but unfortunately there is little evidence that it plays a clinically significant role. There are a number of commercially available tests that purportedly may be employed to identify food intolerances but without strict validation. Such tests include allergen-specific IgG against several foods, sublingual or intradermal provocation tests, electrodermal tests, cytotoxic assays, or others. None of these tests, although fairly popular among concerned patients, is reliable enough to be used as a basis for a dietary therapeutic strategy. On the contrary, diet over-restrictions may lead to nutritional inadequacies or social complexities.

A recently developed test based on direct observation of mucosal reactivity to specific food antigens by endoscopic confocal laser endomicroscopy is encouraging by its simplicity and apparent objectivity [34], but more studies are required to ascertain the practical value of this enticing approach.

\section{Relevance of the Brain-Gut Axis}

Bidirectional communication between brain and gut is essential for the regulation of normal gut function and it seems critical to the development of functional conditions such as IBS [35].

Pain arising from the gut in functional disorders is not simply a matter of stimulated pain afferents by abnormal mechanical or chemical stimuli. Amplified afferent signals (by inflammation, sensitization, and other local factors) result in disturbed pain signaling [36]. Moreover, impaired descending pain modulation further amplifies signals at the central circuits for pain reception and modulation. There is also a link between emotion and cognitive pain modulation implying that stress and anxiety have a major influence on the perception of visceral signals. The main central structures involved are the amygdala, the hypothalamus, and the periaqueductal gray. The main information/control pathways for brain-gut interaction involve both the autonomic nervous system and the hypothalamus-pituitary-adrenal (HPA) axis. Autonomic nervous system disturbances may influence gut reflex activity, perception, and extraintestinal manifestations, common in IBS and other functional gut disorders. Moreover, anxiety/depression appears to facilitate gut inflammation [37] closing the neuroinflammatory brain-gut-brain loop.

Stress is increasingly recognized as an important epidemiological and pathophysiological player in IBS and other functional disorders. Both early stressful events and current chronic stress are important. Thus, early adverse life events may produce permanent epigenetic changes in both the central and enteric nervous systems and also sensitize the HPA axis [38]. Abuse may impact upon central mechanisms of pain modulation, affect, and attention. For instance, we know that hippocampal suppressed neurogenesis induced by stress reduces detection of novel experiences and predisposes to depression [39]. Conversely, brain-derived neurotrophic factor (BDNF) and Bcl-2 promote precursor differentiation resulting in mood 
improvement and pain attenuation. In this context, the HPA system does play a significant role. Brain structures such as the amygdala act as a link between emotional arousal and corticotropin-releasing factor (CFR)-dependent activation of the HPA. In rodents, for instance, increased CRF and urocortin I induce anxiety-like behavior and increase gut sensitivity and motility. In humans, experimental administration of corticotropin-releasing hormone appears to reproduce intestinal effects of experimental stress [40].

The information compiled above leads to the conclusion than abdominal pain, a key feature of IBS, results from a combination of disturbed peripheral pain signaling and disturbed emotional and cognitive pain modulation. As Drossman [41] has pointed out, functional disorders are placed within the range that spans from illness (personal experience of a medical condition) to disease (abnormalities in structure and function of organs and tissues). In a given patient symptom, criteria are insufficient to establish the precise location within the spectrum; however, when the physician carefully takes into consideration the whole clinical picture, the relative weight of central and peripheral mechanisms may be ascertained and help in choosing well among available therapeutic options.

\section{Disturbed Bile Acid Homeostasis}

Chronic diarrhea may be induced by excess bile acids in the colon that stimulate motility and mucus production. Bile acid malabsorption may be observed after ileal resection, cholecystectomy, and there is also an idiopathic form induced by bile acid overproduction in the liver [42]. Bile acid malabsorption has been observed in some patients with IBS-D and postulated as a pathogenetic mechanism for their condition [43-45].

\section{CURRENT TREATMENT APPROACHES FOR IBS}

Taking into consideration the various potential mechanisms involved in the pathogenesis of IBS that we have succinctly reviewed above, one may schematically classify treatment options as shown below and summarized in Table 2:

- Treatments based on diet modifications.

- Treatments directed towards normalization of microbiome ecology.

- Treatments directed against mucosal inflammation.

- Treatments aiming to normalize intestinal motility.

- Treatments directed to correct disturbances along the brain-gut axis and its regulatory pathways.

- Treatment of colonic bile acid overload.

\section{Diet Modifications}

Diet manipulations are a potential therapeutic measure that has been quickly and broadly assumed by the general consumer market without complete understanding of the mechanism of action, criteria for selection of appropriate candidates for dietary adjustments, or guidance for long-term use. Initially, diet recommendations were based on conventional "common sense" and tradition: regular meals, well chewed, avoidance of alcohol, coffee, spices, and greasy foods, etc. Other approaches to dietary control of IBS symptomatology merit consideration. A particularly challenging aspect is the potential effect of bioactive chemicals present in food such as caffeine, histamine, 
Table 2 Current treatment approaches in IBS

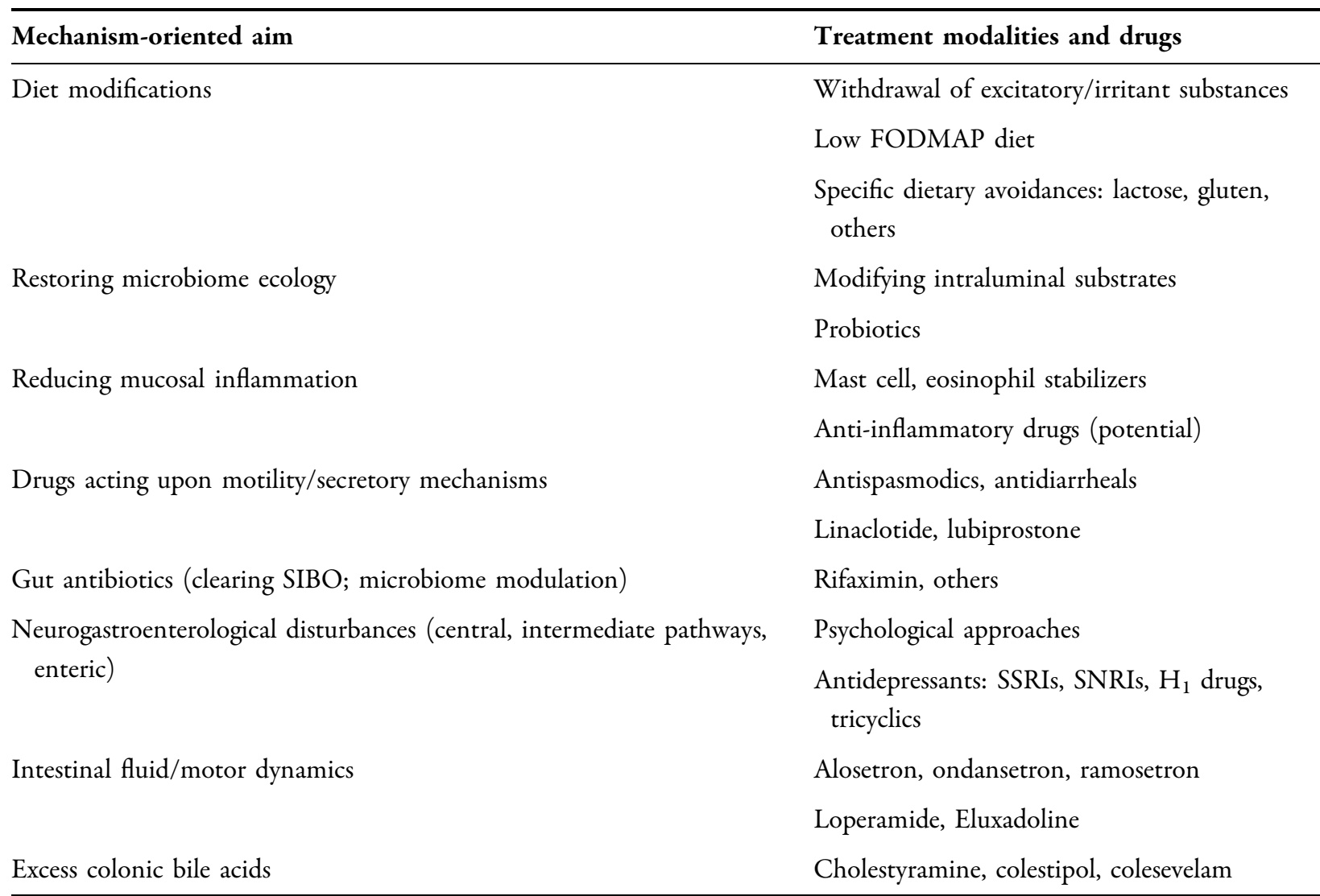

SIBO small intestine bacterial overgrowth, SNRI serotonin-norepinephrine reuptake inhibitor, SSRI selective serotonin reuptake inhibitor

salicylates, and monosodium glutamate to cite a few. Unfortunately it is difficult to design diets that exclude food additives and food chemicals because these industrial molecules are widespread, and highly restricted diets could potentially lead to nutritional inadequacies.

A preeminent antifermentation diet is the so-called low FODMAP diet. The key principle behind this diet is to reduce the intake of poorly absorbed short chain carbohydrates and fermentable vegetables. These food products generate osmotically active substances, generate short chain fatty acids, and increase gas production all of which are poorly tolerated by IBS patients [46-48]. Clinical supporting evidence includes a recent randomized controlled trial by Halmos et al. [49] showing that placement of IBS patients on a FODMAP-restricted diet for 3 weeks significantly improves their symptoms and well-being. Interestingly, adding gluten restriction to patients with non-celiac gluten intolerance does not further improve the response to low FODMAP.

A strict low FODMAP diet is not easy to sustain in the long term, which may account for partial or short-term responses outside controlled trials [50], but it is not a nutrient-deficient diet and fulfills current nutritional standards. However, a low FODMAP diet affects the gut microbiota and we ignore the potential long-term consequences 
of this induced change. Thus, exclusion diets as a general principle should be used for as short a time as possible [51]. Furthermore, others have pointed out that time-honored dietary recommendations for patients with chronic digestive symptoms such as small meals, reduced caffeine, reduced fat, and excess fiber, plus avoidance of well-known gas-producing foodstuffs may achieve similar results as a low FODMAP diet and are easier for patients to grasp and adhere to [52].

\section{Restoring Microbiome Ecology}

There are a substantial number of appealing candidates to influence microbiome ecology for therapeutic intervention in IBS. However, solid proof of significant beneficial action remains elusive for many of the proposed agents. The chief categories are (a) probiotics, i.e., optimal natural bacterial strains (perhaps genetically modified strains in the future); (b) diet manipulations to eliminate or enrich substrates metabolized by bacteria; (c) nonabsorbable antibiotics to actively modify the microbiota ecobalance; and (d) fecal bacteriotherapy that aims at transplanting a foreign colonic flora to re-establish the physiological ecobalance.

Probiotics have become a highly popular medical and paramedical resource for the treatment of IBS and many other functional gastrointestinal disorders. The easy accessibility, fashionability, and perceived safety of probiotics have led to widespread use without much medical modulatory influence or regulatory control. Currently, it is not possible to ascertain whether probiotics, as a class, are indeed useful in the management of IBS. As Shanahan and Quigley [24] have recently pointed out, there are many soft claims plaguing probiotics: labels without verification by controlled human studies, substantial gaps between research findings and marketplace claims, unmet label assertions on numbers and type of viable microbes in commercial probiotic products, and also about quantities of bacteria required to obtain health benefits. Finally, in some instances, shelf-life specifications are not displayed. The current situation reflects in real life the challenges of objectively and ethically translating science into consumer value. Scientists make enticing laboratory observations that sometimes are channeled too quickly by the media without sufficient assessment by regulatory bodies and pushed by eager industries into market distribution without clear-cut evidence of efficacy. Some probiotics have been evaluated by well-designed and robust trials and appear to be effective on certain symptoms under some conditions [53-56], but other probiotic trials have been unsuccessful [57-59].

Many probiotics are commercially available and in fact often tried by patients with IBS with or without professional advice. However, the drawbacks pointed out above remain an obstacle to well-founded medical prescription. Yet, some aspects of probiotic therapy are particularly appealing, such as the concept of a microbiota-brain-gut axis [60, 61]. Indeed, under certain conditions probiotic bacteria may modulate behavior and brain biochemistry via the vagus nerve [62] and other neural and endocrine pathways. Moreover, prior data suggest that anxiety/depression facilitate gut inflammation [63] and, conversely, Bifidobacterium longum may reduce brain emotional reactivity in IBS [64]. Thus, probiotic-induced changes in the gut microbiota appear to modify gut neuromotor functions.

These encouraging laboratory data would support a role for probiotics in the treatment 
of IBS, but outcomes of clinical studies remain equivocal and sometimes contradictory. As pointed out earlier, the lack of good biomarkers of healthy microbiota to predict disease has slowed down progress in search for appropriate probiotic therapy. Future progress in this field is likely to produce useful advances.

\section{Acting on Gut Mucosal Inflammation}

Intestinal inflammation, be it post-infectious, allergic, or idiopathic, is a recognizable feature, especially in IBS-D but also in other IBS subgroups. Some years ago, it was observed that chronic users of steroids were at a reduced risk for developing IBS [65]. Disappointingly, however, additional studies showed that treatment of patients with post-infectious IBS with prednisolone was not clinically efficacious [66], and two recent trials of mesalazine treatment in IBS yielded negative outcomes $[67,68]$. On the other hand, a small trial with the mast cell stabilizer ketotifen has produced encouraging results [69]. The negative results of the mesalazine trials have somewhat dampened the enthusiasm for the concept of anti-inflammatory therapy in IBS; however, as Törnblom and Simren [70] have recently pointed out, given the current obstacles to select potentially responsive IBS subgroups and lack of consensus on primary endpoints to measure, it may be premature to give up on these anti-inflammatory therapy approaches.

\section{Drugs Acting on Intestinal Motor and/ or Secretory Function}

Traditionally this has been the preferred therapeutic route beginning with classical antispasmodics, laxatives of different sorts, and antidiarrheal agents such as loperamide. Many of these agents are currently in use by practitioners around the world to help control pain and diarrhea in patients with IBS.

Most antispasmodics are anticholinergic agents that exhibit some effect, mostly documented in vitro, reducing spasmodic contractions of bowel muscles. They have not been rigorously evaluated by well-designed clinical trials. Thus, their clinical efficacy remains in question despite over 50 years of use for pain control and diarrhea. Peppermint oil is an antispasmodic agent with a different mechanism of action (reduces influx of calcium in smooth muscle cells). Other agents sharing this mechanism of action include otilonium bromide and mebeverine. This latter class of agents do not have anticholinergic side effects.

However, newer and more refined agents have come along. Linaclotide is a molecule that promotes intestinal secretion and appears to inhibit visceral pain signals. It is particularly useful for the treatment of IBS-C. However, the higher capsule dosage of linaclotide $(290 \mu \mathrm{g})$, showing antinociceptive visceral effects in the clinical trials, carries a relatively high probability of inducing annoying diarrhea. Likewise, lubiprostone, a marketed drug that acts by promoting water secretion in the upper intestine, also appears to be useful for the treatment of IBS-C.

\section{Rifaximin and Other Antibiotics in IBS}

Nonabsorbable antibiotics represent a different approach to modifying the microbiota for therapeutic purposes. They have been proposed on the basis of two main concepts: control of small bowel bacterial overgrowth and modification of colonic microbiota.

Broad-spectrum antibiotics have been evaluated for the treatment of IBS largely on the basis of presumed SIBO [21]. Rifaximin has been favored on the basis of its 
nonabsorbability and good safety profile, as well as empirical evidence of efficacy provided by various trials [71, 72]. It appears that 10-14 days of treatment with rifaximin (800-1650 mg/day) may improve global IBS symptoms and IBS-related bloating. The improvement persists for several weeks. Efficacy has been better documented for patients with IBS-D than for other IBS subgroups. The mechanism of action of rifaximin in patients with IBS improved by this modality of treatment remains unclear. Rifaximin shows strong antibiotic action against bacterial species commonly found in SIBO; however, as indicated earlier, the symptomatic benefit produced by rifaximin could also be mediated through modulation of the colonic microbiome ecosystem. With rifaximin therapy, hydrogen gut production generally diminishes in association with symptomatic improvement. Rifaximin shows greater efficacy in relieving IBS symptoms than previously tried broad-spectrum antibiotics, possibly on account of the higher local concentrations achieved by the unabsorbable rifaximin molecule [73]. Other potential mechanisms, albeit not fully evaluated, include putative anti-inflammatory actions of rifaximin and modifying effects on microbiota function including bacterial virulence and bacterial metabolic activity that in turn may lead to normalization of intestinal motility and attenuation of visceral hyperalgesia [74].

\section{Neurogastroenterological Therapeutic Agents}

As pointed out earlier, a number of key neurotransmitters, neural circuits, and integrated brain, spinal, and ganglia structures probably contribute to the generation of pain and associated symptoms in patients with IBS. Unfortunately we are often unable to determine at which level or levels along the brain-gut axis the most relevant disturbances are occurring. It seems likely that the role of central versus peripheral regulatory dysfunction varies considerably among individual patients with IBS. Generally speaking, the more severe and refractory the pain component is, the greater the participation of the brain is in the pathogenesis of symptoms [41]. Neuropharmacological treatments are to some extent hampered by uncertainty as to the mechanisms involved. Thus, best results are often obtained by drugs or combination of drugs that act simultaneously upon central and peripheral drug targets.

Psychological approaches also appear to be effective, although highly dependent on time and operator skills. Clinicians should be aware of the remarkably high placebo response rates (35-70\%) observed in most controlled trials of IBS and other functional-type conditions. In practice, many physicians take advantage of these positive placebo effects although unfortunately they tend to be short lived. Some lifestyle recommendations can probably be included among psychological approaches. Other more formal and structured modalities of psychotherapy have been employed but being time consuming and available only at specialized centers has limited their general use. Hypnotherapy and behavioral modification techniques must be included in this category.

Antidepressant drugs have a role in the management of IBS. These agents have several principal objectives: to attenuate visceral hypersensitivity, to regulate gastrointestinal motility, to improve mood and reduce anxiety, to control anorexia, and to improve sleep. These objectives are best prioritized by carefully assessing first the clinical features perceived in a given patient. This facilitates 
taking advantage of pharmacological properties of different drugs or combination of drugs to maximize favorable effects.

Control of abdominal pain via attenuation of visceral hypersensitivity is a major therapeutic objective in most patients. Modern antidepressants with combined inhibitory affinity for serotonin-norepinephrine reuptake inhibitors (SNRI) such as venlafaxine and duloxetine are generally preferred to pure serotonin reuptake inhibitors (SRI) like fluoxetine, paroxetine, or sertraline. Classic tricyclic antidepressants such as amitriptyline, nortriptyline, or desipramine can also be quite effective for pain-relieving purposes but tend to induce constipation (thus, they are more useful for IBS-D). However, other less favorable eye and prostate side effects of the older tricyclic drugs may also limit their use.

Some antidepressants with histamine $\mathrm{H}_{1}$ receptor affinity tend to produce somnolence and weight gain (i.e., mirtazapine). These properties may be useful to help some patients obtain better sleep and to stimulate their appetite but may be inconvenient to other patients. Side effects of some antidepressants such as sexual dysfunction, arrhythmia, restlessness, and orthostatic hypotension may pose difficulties in some cases and also limit their usefulness. Furthermore, it has been pointed out that side effects tend to be more common and limiting when antidepressants are used for IBS than when used for major depression, perhaps reflecting the peculiarities of functional-type patients. As a rule, off-label treatment of IBS with antidepressants involves much lower doses than for overt psychiatric disorders. However, some patients with IBS are truly affected by anxiety/depression and may benefit from standard doses of these drugs.

\section{Agents that Modify Colonic Fluid and Motor Dynamics}

Alosetron, a serotonin $5 \mathrm{HT}_{3}$ antagonist, was introduced in the late 1990s in the USA for the treatment of IBS-D. Subsequently it was withdrawn from the market after reports of patients who developed severe constipation and/or ischemic colitis and then reintroduced at a lower recommended dose under a risk management program. Ondansetron, an antiemetic agent with a related pharmacodynamic profile, appears to be an effective substitute at doses as low as $2 \mathrm{mg}$ every other day. Ramosetron, another $5 \mathrm{HT}_{3}$ receptor antagonist, is showing encouraging outcomes in recent trials [75].

Eluxadoline, recently approved in the USA for the treatment of IBS-D, is a $\mu$-opioid receptor agonist/ $\delta$-opioid receptor antagonist/k-receptor agonist. The three major receptors $(\mathrm{k}, \delta$, and $\mu)$ are found in the enteric nervous system, smooth muscle cells, and pacemaker cells of the gastrointestinal tract. Loperamide, a well-known antidiarrheal agent, is a $\mu$-opioid receptor antagonist with limited capacity to cross the blood-brain barrier. Its main drawback in patients with IBS-D is that it tends to induce excess constipation. Eluxadoline's $\mu$-agonist affect probably accounts for its antidiarrheal action, whereas its $\delta$-opioid receptor antagonism accounts for is modulatory effects on visceral hypersensitivity and attenuates the strong motility inhibitory effect of $\mu$-agonism [76]. Thus, it does not exhibit the strong constipating effect of loperamide. Furthermore, the $\delta$ agonist action of eluxadoline appears to prevent the development of tolerance. Eluxadoline may also inhibit neurogenically mediated bowel 
secretion, further controlling diarrhea. Clinical phase III studies have been completed on patients with IBS-D using either 75 or $100 \mathrm{mg}$ tablets of eluxadoline twice daily for 26 weeks. Results showed significant improvement in stool frequency and consistency, as compared to placebo, as well as improvement in abdominal pain for the entire duration of the study (26 weeks) though only for the higher $100 \mathrm{mg}$ twice-daily dose. Thus, a standard dosage of $100 \mathrm{mg}$ twice daily has been recommended for general practice. The safety profile of eluxadoline is reasonably acceptable with the most common adverse effects being constipation (usually mild), nausea and vomiting or abdominal pain. Some concern has been raised for the rare $(<1 \%)$ but significant event of sphincter of Oddi spasm with elevated liver enzymes and/or mild pancreatitis. All cases with pancreatitis had absent gallbladders and, therefore, a lower dose of $75 \mathrm{mg}$ twice daily may be more appropriate for previously cholecystectomized patients. Also, patients prone to use excess alcohol should be warned of the increased risk of pancreatitis with $\mu$-agonist agents such as eluxadoline.

\section{Bile Acid Sequestrants}

Cholestyramine is a nondigestible resin that binds to bile acids in the intestine and prevents their irritating colonic effects while increasing their excretion in feces. Cholestyramine has been widely employed in various diarrheal conditions associated with excess colonic bile acids. It appears to be effective in patients with IBS-D with elevated bile acid excretion [44]. Cholestyramine is unpalatable, which decreases patient compliance and may induce uncomfortable symptoms on its own such as constipation, flatulence, bloating, and others, but these are usually minor [77]. Colestipol is a somewhat more palatable bile acid sequestrant with similar action. Colesevelam is the latest sequestrant to be developed and it is available in tablet form which increases its acceptability [78]. Bile acid synthesis increases during colesevelam therapy and appears to compensate for increased fecal bile acid loss, hence preventing fat malabsorption in the proximal small bowel [43]. A trial of bile acid sequestrants may be appropriate for patients with IBS-D.

\section{ACKNOWLEDGMENTS}

No funding or sponsorship was received for publication of this article. All named authors meet the International Committee of Medical Journal Editors (ICMJE) criteria for authorship for this manuscript, take responsibility for the integrity of the work as a whole, and have given final approval for the version to be published.

Disclosures. Juan R. Malagelada and Carolina Malagelada have nothing to disclose.

Compliance with Ethics Guidelines. This article is based on previously conducted studies and does not involve any new studies of human or animal subjects performed by any of the authors.

Open Access. This article is distributed under the terms of the Creative Commons Attribution-NonCommercial 4.0 International License (http://creativecommons.org/licenses/ by-nc/4.0/), which permits any noncommercial use, distribution, and reproduction in any medium, provided you give appropriate credit to the original author(s) and the source, provide a link to the Creative Commons license, and indicate if changes were made. 


\section{REFERENCES}

1. Sperber AD, Dumitrascu D, Fukudo S, et al. The global prevalence of IBS in adults remains elusive due to the heterogeneity of studies: a Rome Foundation working team literature review. Gut 2016. doi:10.1136/gutjnl-2015-311240.

2. Russo MW, Gaynes BN, Drossman DA. A national survey of practice patterns of gastroenterologists with comparison to the past two decades. J Clin Gastroenterol. 1999;29(4):339-43.

3. Card TR, Siffledeen J, Fleming KM. Are IBD patients more likely to have a prior diagnosis of irritable bowel syndrome? Report of a case-control study in the General Practice Research Database. United Eur Gastroenterol J. 2014;2(6):505-12.

4. Card TR, Siffledeen J, West J, Fleming KM. An excess of prior irritable bowel syndrome diagnoses or treatments in celiac disease: evidence of diagnostic delay. Scand J Gastroenterol. 2013;48(7):801-7.

5. Quigley EM, Shanahan F. The language of medicine: words as servants and scoundrels. Clin Med (Lond). 2009;9(2):131-5.

6. Longstreth GF, Thompson WG, Chey WD, Houghton LA, Mearin F, Spiller RC. Functional bowel disorders. Gastroenterology. 2006;130(5):1480-91.

7. Chang L, Berman S, Mayer EA, et al. Brain responses to visceral and somatic stimuli in patients with irritable bowel syndrome with and without fibromyalgia. Am J Gastroenterol. 2003;98(6):1354-61.

8. Ross SE. "Memes" as infectious agents in psychosomatic illness. Ann Intern Med. 1999;131(11):867-71.

9. Chadwick VS, Chen W, Shu D, et al. Activation of the mucosal immune system in irritable bowel syndrome. Gastroenterology. 2002;122(7):1778-83.

10. Cremon C, Gargano L, Morselli-Labate AM, , et al. Mucosal immune activation in irritable bowel syndrome: gender-dependence and association with digestive symptoms. Am J Gastroenterol. 2009;104(2):392-400.

11. Matricon J, Meleine M, Gelot A, et al. Review article: associations between immune activation, intestinal permeability and the irritable bowel syndrome. Aliment Pharmacol Ther. 2012;36(11-12):1009-31.

12. O'Malley D. Immunomodulation of enteric neural function in irritable bowel syndrome. World J Gastroenterol. 2015;21(24):7362-6.
13. Sundin J, Rangel I, Kumawat AK, Hultgren-Hornquist E, Brummer RJ. Aberrant mucosal lymphocyte number and subsets in the colon of post-infectious irritable bowel syndrome patients. Scand J Gastroenterol. 2014;49(9):1068-75.

14. Barbara G, Wang B, Stanghellini V, et al. Mast cell-dependent excitation of visceral-nociceptive sensory neurons in irritable bowel syndrome. Gastroenterology. 2007;132(1):26-37.

15. Martinez C, Lobo B, Pigrau $M$, et al. Diarrhoea-predominant irritable bowel syndrome: an organic disorder with structural abnormalities in the jejunal epithelial barrier. Gut. 2013;62(8):1160-8.

16. Bashashati M, Rezaei N, Shafieyoun A, et al. Cytokine imbalance in irritable bowel syndrome: a systematic review and meta-analysis. Neurogastroenterol Motil. 2014;26(7):1036-48.

17. Cocciolillo S, Collins SM. The long-term functional consequences of acute infectious diarrhea. Curr Opin Gastroenterol. 2016;32(1):1-6.

18. Schwille-Kiuntke J, Mazurak N, Enck P. Systematic review with meta-analysis: post-infectious irritable bowel syndrome after travellers' diarrhoea. Aliment Pharmacol Ther. 2015;41(11):1029-37.

19. Wouters MM, Van Wanrooy S, Nguyen A, et al. Psychological comorbidity increases the risk for postinfectious IBS partly by enhanced susceptibility to develop infectious gastroenteritis. Gut 2015. doi:10.1136/gutjnl-2015-309460.

20. Spreadbury I, Ochoa-Cortes F, Ibeakanma C, Martin N, Hurlbut D, Vanner SJ. Concurrent psychological stress and infectious colitis is key to sustaining enhanced peripheral sensory signaling. Neurogastroenterol Motil. 2015;27(3):347-55.

21. Pimentel M, Chow EJ, Lin HC. Eradication of small intestinal bacterial overgrowth reduces symptoms of irritable bowel syndrome. Am J Gastroenterol. 2000;95(12):3503-6.

22. Di Stefano M, Corazza GR. The rationale for antibiotics in IBS. Am J Gastroenterol. 2008;103(10):2652.

23. Bratten JR, Spanier J, Jones MP. Lactulose breath testing does not discriminate patients with irritable bowel syndrome from healthy controls. Am J Gastroenterol. 2008;103(4):958-63.

24. Shanahan F, Quigley EM. Manipulation of the microbiota for treatment of IBS and IBD-challenges and controversies. Gastroenterology. 2014;146(6):1554-63. 
25. $\mathrm{Yu}$ D, Cheeseman F, Vanner S. Combined oro-caecal scintigraphy and lactulose hydrogen breath testing demonstrate that breath testing detects oro-caecal transit, not small intestinal bacterial overgrowth in patients with IBS. Gut. 2011;60(3):334-40.

26. Rajilic-Stojanovic M, de Vos WM. The first 1000 cultured species of the human gastrointestinal microbiota. FEMS Microbiol Rev. 2014;38(5):996-1004.

27. Miquel S, Martin $\mathrm{R}$, Lashermes $\mathrm{A}$, et al. Anti-nociceptive effect of Faecalibacterium prausnitzii in non-inflammatory IBS-like models. Sci Rep. 2016;6. doi:10.1038/srep19399.

28. Wegner A, Banaszkiewicz A, Kierkus J, et al. Effectiveness of Lactobacillus reuteri in the treatment of functional constipation in children: a randomized, double-blind, placebo-controlled, multicenter trial. United Eur Gastroenterol J. 2015;3(5S):A20.

29. Itan Y, Jones BL, Ingram CJ, Swallow DM, Thomas MG. A worldwide correlation of lactase persistence phenotype and genotypes. BMC Evol Biol. 2010;10:36.

30. Casellas F, Aparici A, Casaus M, Rodriguez P, Malagelada JR. Subjective perception of lactose intolerance does not always indicate lactose malabsorption. Clin Gastroenterol Hepatol. 2010;8(7):581-6.

31. Rumessen JJ, Gudmand-Høyer E. Absorption capacity of fructose in healthy adults. Comparison with sucrose and its constituent monosaccharides. Gut 1986;27:1161-8.

32. Madsen JL, Linnet J, Rumessen JJ. Effect of nonabsorbed amounts of a fructose-sorbitol mixture on small intestinal transit in healthy volunteers. Dig Dis Sci. 2006;51(1):147-53.

33. Murray K, Wilkinson-Smith V, Hoad C, et al. Differential effects of FODMAPs (fermentable oligo-, di-, mono-saccharides and polyols) on small and large intestinal contents in healthy subjects shown by MRI. Am J Gastroenterol. 2014;109(1):110-9.

34. Fritscher-Ravens A, Schuppan D, Ellrichmann M, et al. Confocal endomicroscopy shows food-associated changes in the intestinal mucosa of patients with irritable bowel syndrome. Gastroenterology. 2014;147(5):1012-20.

35. Koloski NA, Jones M, Kalantar J, Weltman M, Zaguirre J, Talley NJ. The brain-gut pathway in functional gastrointestinal disorders is bidirectional: a 12-year prospective population-based study. Gut. 2012;61(9):1284-90.

36. Buhner S, Li Q, Vignali S, et al. Activation of human enteric neurons by supernatants of colonic biopsy specimens from patients with irritable bowel syndrome. Gastroenterology. 2009;137(4):1425-34.

37. Ghia JE, Blennerhassett P, Collins SM. Impaired parasympathetic function increases susceptibility to inflammatory bowel disease in a mouse model of depression. J Clin Invest. 2008;118(6):2209-18.

38. Dieleman GC, Huizink AC, Tulen JH, et al. Alterations in HPA-axis and autonomic nervous system functioning in childhood anxiety disorders point to a chronic stress hypothesis. Psychoneuroendocrinology. 2015;51:135-50.

39. Perera TD, Park S, Nemirovskaya Y. Cognitive role of neurogenesis in depression and antidepressant treatment. Neuroscientist. 2008;14(4):326-38.

40. Pritchard SE, Garsed KC, Hoad CL, et al. Effect of experimental stress on the small bowel and colon in healthy humans. Neurogastroenterol Motil. 2015;27(4):542-9.

41. Drossman DA. Redux: do little bellyachers grow up to become big bellyachers? Clin Gastroenterol Hepatol. 2014;12(12):2033-6.

42. Walters JR. Bile acid diarrhoea and FGF19: new views on diagnosis, pathogenesis and therapy. Nat Rev Gastroenterol Hepatol. 2014;11(7):426-34.

43. Camilleri M, Busciglio I, Acosta A, et al. Effect of increased bile acid synthesis or fecal excretion in irritable bowel syndrome-diarrhea. Am J Gastroenterol. 2014;109(10):1621-30.

44. Bajor A, Tornblom H, Rudling M, Ung KA, Simren M. Increased colonic bile acid exposure: a relevant factor for symptoms and treatment in IBS. Gut. 2015;64(1):84-92.

45. Aziz I, Mumtaz S, Bholah H, Chowdhury FU, Sanders DS, Ford AC. High prevalence of idiopathic bile acid diarrhea among patients with diarrhea-predominant irritable bowel syndrome based on Rome III criteria. Clin Gastroenterol Hepatol. 2015;13(9):1650-5.

46. Gibson PR, Shepherd SJ. Evidence-based dietary management of functional gastrointestinal symptoms: the FODMAP approach. J Gastroenterol Hepatol. 2010;25(2):252-8.

47. Shepherd SJ, Lomer MC, Gibson PR. Short-chain carbohydrates and functional gastrointestinal disorders. Am J Gastroenterol. 2013;108(5):707-17. 
48. De GR, Volta U, Gibson PR. Sensitivity to wheat, gluten and FODMAPs in IBS: facts or fiction? Gut. 2016;65(1):169-78.

49. Halmos EP, Power VA, Shepherd SJ, Gibson PR, Muir JG. A diet low in FODMAPs reduces symptoms of irritable bowel syndrome. Gastroenterology. 2014;146(1):67-75.

50. Shepherd SJ, Gibson PR. Fructose malabsorption and symptoms of irritable bowel syndrome: guidelines for effective dietary management. J Am Diet Assoc. 2006;106(10):1631-9.

51. Lomer MC. Review article: the aetiology, diagnosis, mechanisms and clinical evidence for food intolerance. Aliment Pharmacol Ther. 2015;41(3):262-75.

52. Bohn L, Storsrud S, Liljebo T, et al. Diet low in FODMAPs reduces symptoms of irritable bowel syndrome as well as traditional dietary advice: a randomized controlled trial. Gastroenterology. 2015;149(6):1399-407.

53. Whorwell PJ, Altringer L, Morel J, et al. Efficacy of an encapsulated probiotic Bifidobacterium infantis 35624 in women with irritable bowel syndrome. Am J Gastroenterol. 2006;101(7):1581-90.

54. Eskesen D, Jespersen L, Michelsen B, Whorwell PJ, Muller-Lissner S, Morberg CM. Effect of the probiotic strain Bifidobacterium animalis subsp. lactis, BB-12(R), on defecation frequency in healthy subjects with low defecation frequency and abdominal discomfort: a randomised, double-blind, placebo-controlled, parallel-group trial. Br J Nutr. 2015;114(10):1638-46.

55. Sisson G, Ayis S, Sherwood RA, Bjarnason I. Randomised clinical trial: a liquid multi-strain probiotic vs. placebo in the irritable bowel syndrome-a 12 week double-blind study. Aliment Pharmacol Ther. 2014;40(1):51-62.

56. Yoon JS, Sohn W, Lee OY, et al. Effect of multispecies probiotics on irritable bowel syndrome: a randomized, double-blind, placebo-controlled trial. J Gastroenterol Hepatol. 2014;29(1):52-9.

57. Sondergaard B, Olsson J, Ohlson K, Svensson U, Bytzer P, Ekesbo R. Effects of probiotic fermented milk on symptoms and intestinal flora in patients with irritable bowel syndrome: a randomized, placebo-controlled trial. Scand J Gastroenterol. 2011;46(6):663-72.

58. Roberts LM, McCahon D, Holder R, Wilson S, Hobbs FD. A randomised controlled trial of a probiotic 'functional food' in the management of irritable bowel syndrome. BMC Gastroenterol. 2013;13:45.

59. Stevenson C, Blaauw R, Fredericks E, Visser J, Roux S. Randomized clinical trial: effect of Lactobacillus plantarum $299 \mathrm{v}$ on symptoms of irritable bowel syndrome. Nutrition. 2014;30(10):1151-7.

60. Catanzaro R, Anzalone M, Calabrese F, et al. The gut microbiota and its correlations with the central nervous system disorders. Panminerva Med. 2015;57(3):127-43.

61. Mayer EA, Savidge T, Shulman RJ. Brain-gut microbiome interactions and functional bowel disorders. Gastroenterology. 2014;146(6):1500-12.

62. Bravo JA, Forsythe $\mathrm{P}$, Chew MV, et al. Ingestion of Lactobacillus strain regulates emotional behavior and central GABA receptor expression in a mouse via the vagus nerve. Proc Natl Acad Sci USA. 2011;108(38):16050-5.

63. Ghia JE, Park AJ, Blennerhassett P, Khan WI, Collins SM. Adoptive transfer of macrophage from mice with depression-like behavior enhances susceptibility to colitis. Inflamm Bowel Dis. 2011;17:1479-89.

64. Pinto-Sanchez MI, Hall GB, Ghajar K, et al. Bifidobacterium longum NCC3001 improves depression and reduces brain emotional reactivity in patients with irritable bowel syndrome (IBS): a randomized, double blind, placebo-controlled trial. United Eur Gastroenterol J. 2015;3(5S):A53.

65. Huerta C, Garcia Rodriguez LA, Wallander MA, Johansson S. Users of oral steroids are at a reduced risk of developing irritable bowel syndrome. Pharmacoepidemiol Drug Saf. 2003;12(7):583-8.

66. Dunlop SP, Jenkins D, Neal KR, et al. Randomized, double-blind, placebo-controlled trial of prednisolone in post-infectious irritable bowel syndrome. Aliment Pharmacol Ther. 2003;18(1):77-84.

67. Lam C, Tan W, Leighton M, et al. A mechanistic multicentre, parallel group, randomised placebo-controlled trial of mesalazine for the treatment of IBS with diarrhoea (IBS-D). Gut. 2016;65(1):91-9.

68. Barbara G, Cremon C, Annese V, et al. Randomised controlled trial of mesalazine in IBS. Gut. 2016;65(1):82-90.

69. Klooker TK, Braak B, Koopman KE, et al. The mast cell stabiliser ketotifen decreases visceral hypersensitivity and improves intestinal symptoms in patients with irritable bowel syndrome. Gut. 2010;59:1213-21. 
70. Tornblom $\mathrm{H}$, Simren $\mathrm{M}$. In search for a disease-modifying treatment in irritable bowel syndrome. Gut. 2016;65(1):2-3.

71. Yang J, Lee HR, Low K, Chatterjee S, Pimente M. Rifaximin versus other antibiotics in the primary treatment and retreatment of bacterial overgrowth in IBS. Dig Dis Sci. 2008;53(1):169-74.

72. Menees SB, Maneerattannaporn M, Kim HM, Chey WD. The efficacy and safety of rifaximin for the irritable bowel syndrome: a systematic review and meta-analysis. Am J Gastroenterol. 2012;107(1):28-35.

73. Jiang ZD, Ke S, Palazzini E, Riopel L, Dupont H. In vitro activity and fecal concentration of rifaximin after oral administration. Antimicrob Agents Chemother. 2000;44(8):2205-6.

74. Pimentel M. Review article: potential mechanisms of action of rifaximin in the management of irritable bowel syndrome with diarrhoea. Aliment Pharmacol Ther. 2016;43(Suppl 1):37-49.
75. Fukudo S, Kinoshita Y, Okumura $\mathrm{T}$, et al. Ramosetron reduces symptoms of irritable bowel syndrome with diarrhea and improves quality of life in women. Gastroenterology. 2016;150(2):358-66.

76. Wade PR, Palmer JM, McKenney S, et al. Modulation of gastrointestinal function by MuDelta, a mixed micro opioid receptor agonist/ micro opioid receptor antagonist. Br J Pharmacol. 2012;167(5):1111-25.

77. Fernandez-Banares F, Rosinach M, Piqueras M, et al. Randomised clinical trial: colestyramine vs. hydroxypropyl cellulose in patients with functional chronic watery diarrhoea. Aliment Pharmacol Ther. 2015;41(11):1132-40.

78. Camilleri M, Acosta A, Busciglio I, et al. Effect of colesevelam on faecal bile acids and bowel functions in diarrhoea-predominant irritable bowel syndrome. Aliment Pharmacol Ther. 2015;41(5):438-48. 